\title{
A new test procedure to evaluate the performance of substations for collective heating systems
}

\author{
Robin Baetens ${ }^{1, *}$, and Ivan Verhaert ${ }^{1}$ \\ ${ }^{1}$ University of Antwerp, Faculty of Applied Engineering, Energy and Material in Infrastructure and \\ Buildings, Campus Groenenborger, Groenenborgerlaan 171, 2020 Antwerp, building Z
}

\begin{abstract}
The overall heat demand of a single dwelling, existing out of space heating and domestic hot water production, decreases due to higher insulation rates. Because of this, investing in efficient and renewable heat generation becomes less interesting. Therefore, to incorporate renewables or residual heat on a larger scale, district heating or collective heating systems grow in importance. Within this set-up, the substation is responsible for the interaction between local demand for comfort and overall energy performance of the collective heating system. Many different configurations of substations exist, which influence both local comfort and central system performance. Next to that, also hybrids exist with additional local energy input. To evaluate performance of such substations, a new experimental-based test procedure is developed in order to evaluate these different aspects, characterized by the two roles a substation has, namely as heat generator and as heat consumer. The advantage of this approach is that an objective comparison between individual and central systems regarding performance on delivering local comfort can be executed experimentally. The lab set-up consists out of three different subsystems, namely the central system, the domestic hot water consumption and the local space heating. The central system can work with different temperature regimes and control strategies, as these aspects have proven to have the largest influence on actual performance. The domestic hot water system is able to generate similar tap profiles according to eco-design regulation for domestic hot water generation. The space heating system is able to demand a modular heat load.
\end{abstract}

* Corresponding author: robin.baetens@uantwerpen.com 


\section{Introduction}

Today, we face great challenges concerning energy. The consequences of climate change and the depletion of fossil fuels like oil and natural gas, have led to following commitments of the EU, compared to 1990 [1].

- increasing the share of renewable energy sources by $20 \%$ in 2020 and by $27 \%$ in 2030

- increasing energy efficiency by $20 \%$ in 2020 and by $27 \%$ in 2030

- reducing emissions by $20 \%$ in 2020 , by $40 \%$ in 2030 and by $80-95 \%$ in 2050

As heat is responsible for about $40-50 \%$ of our energy usage, increasing the overall efficiency of this heat utilization is crucial in order to achieve these objectives. A first step should always be reducing demand, e.g. by improving the insulation rate of our building stock. Secondly, the heat generation has to become more sustainable by increasing the share of renewables and/or its efficiency. However, with a decreasing heat demand, investments in efficient local heat generators become less beneficial. From this point of view a, rather 'old', heating design strategy becomes an option again, namely collective heating systems or district heating.

It has been shown that if the potential of district heating will be fully exploited, the target of 2050 of the EU to reduce energy consumption can be realized, even with a cost reduction of $15 \%$ for the heating and cooling demands of buildings [2]. If the combustion systems at the end users can be eliminated, this will also mean a safer heat production (no more gas in city and buildings) and less space occupation. On top of that, local pollutants will decrease, which will have a positive effect on air quality in city areas and hence health of the inhabitants [3]. Next, district heating enables the use of excess heat from e.g. industrial processes. With the city of Antwerp as the example par excellence, it is not surprising, Persson et al. (2014) found a very high spatial correlation of $46 \%$ between heat excess and heat demand in the EU27. Also, in terms of storage, thermal grids have an advantage with the inexpensive storage methods for heat (compared to e.g. batteries in an electric grid).

With respect to the acceptance of collective heating systems, worries arise whether collective heating systems are effective in reaching local comfort demand. The substation is the link between this local comfort demand and energy efficient collective heating system.

As can be expected, the type of substation will have a large influence on both local comfort (meeting heat demand with little time delay) and on energy efficiency of the district heating network (ensuring and/or allowing low distribution temperatures and efficient control strategies).

A diversity of products are available on the market. Therefore, it is necessary to develop a proper test procedure, which addresses the different KPIs a substation should meet, allowing a proper comparison between different types and if possible to compare them with local heat generation.

In this paper a lab set-up is built which allows to execute different test procedures for substations. Special attention is paid to evaluate dynamic behaviour in order to gain the necessary parameters to model different kinds of substations within a heating network. Next to that, to allow comparison with local heat generators the set-up is built in such a way, that they can be tested under the same circumstances.

After the design of a lab set-up, different test procedures are evaluated. 


\section{Description of the test facility}

Originally a test facility was built to test heat generators according to NBN EN 13203-1 [5] and 2 [6]. Acknowledging the lack of in-depth test results for substations, the test facility was rebuilt and expanded. The present lab set-up consists out of three different subsystems, namely the central system, the domestic hot water consumption and the local space heating. The central system (CS) should be able to simulate behaviour of different kinds of collective heating networks. Therefore, it is crucial that it can work with different temperature regimes and control strategies, as these aspects have been proven to have the largest influence on actual performance. To evaluate end-use comfort, the domestic hot water system (DHW) is able to generate similar tap profiles according to eco-design regulation for domestic hot water generation. The space heating system $(\mathrm{SH})$ is able to demand a modular heat load.

\subsection{Central system}

A small household combi boiler with an approximated output of $30 \mathrm{~kW}$ provides energy to a 1501 buffer. A $0 \ldots 10 \mathrm{~V}$ regulated circulation pump provides different flow rates. Through smart switching of ball valves different pipe lengths varying from $10 \mathrm{~m}$ to $50 \mathrm{~m}$ can be added. These variations simulate the effects of long distant stations and how the supply/return temperature varies over time with a fluctuating thermal load. Based on NBN EN 13203, sensors are placed as close as possible to the station eliminating the need for calculating losses in the central or DHW system. The sensors of each subdivision consist of a rapid response thermometer in each line, a differential pressure transmitter between supply and return, a flow transmitter in the hot line and finally for backup a heat meter installed in the cold line. The combination of high precision temperature and flow sensors allows the calculation of thermal energy flow, backed up by the heat meter, which is more accurate, but has a lower measuring frequency, approximately $16 \mathrm{~s}$ between refreshes compared to $1 \mathrm{~s}$. The measurement of the paired differential pressure offers insight in the pumping losses due to the substation.

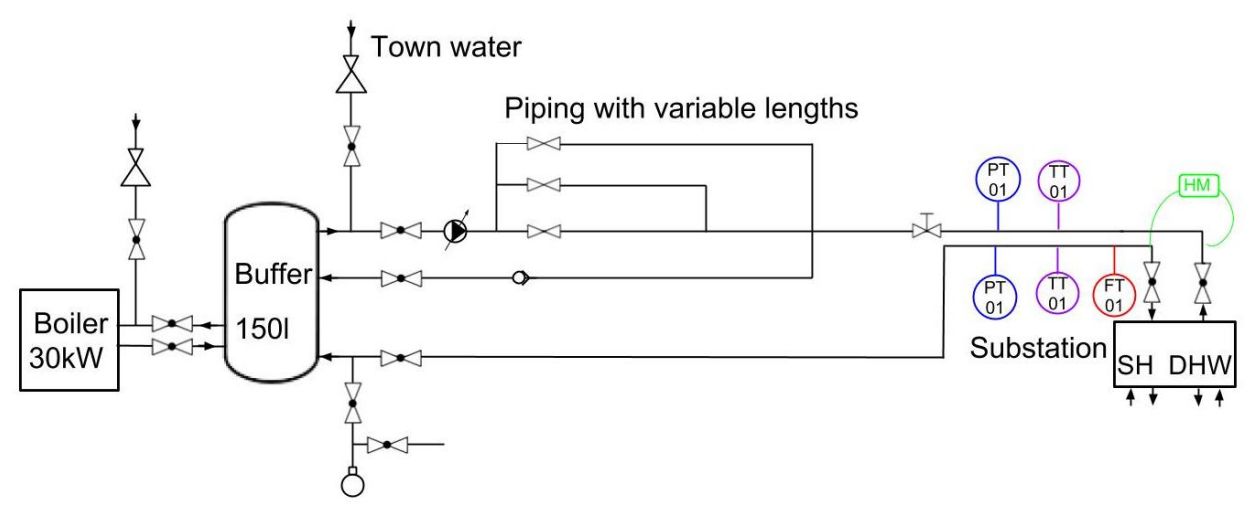

Fig. 1. Primary circuit, heat supply central system. 


\subsection{Domestic hot water consumption}

Using a PLC in the configuration, it is possible to simultaneously read sensor values and drive actuators. Through electromagnetic valves and mechanically adjustable dynamic flow restrictors, a tapping cycle, as described in NBN EN 13203-2 is applied. Besides the energy performance at the selected tapping cycle, also other aspects can be examined referring to the behaviour of the unit at comfort level. For instance when the unit gets temperature shocked, albeit from a cold start or with a constant withdrawal of SH.

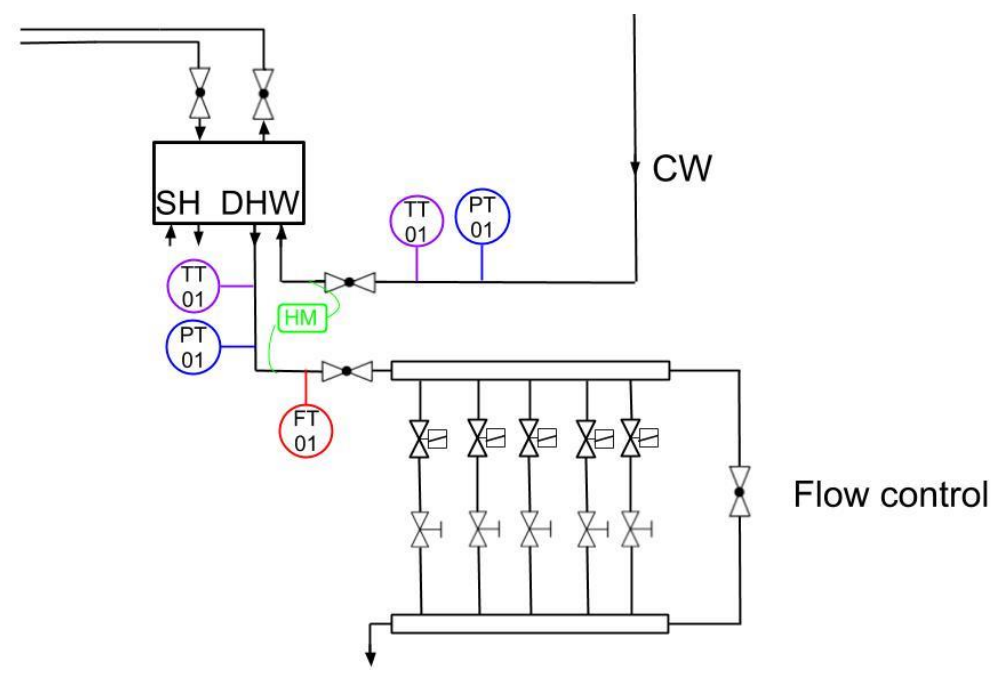

Fig. 2. Secondary circuit, Domestic hot water consumption.

Cold water $(\mathrm{CW})$ needs to be provided at a constant temperature of $10 \pm 2^{\circ} \mathrm{C}$. A 3001 buffer is kept at a temperature close to $10^{\circ} \mathrm{C}$. To maintain a uniform temperature without stratification, a circulation pump is powered when no withdrawal is made.

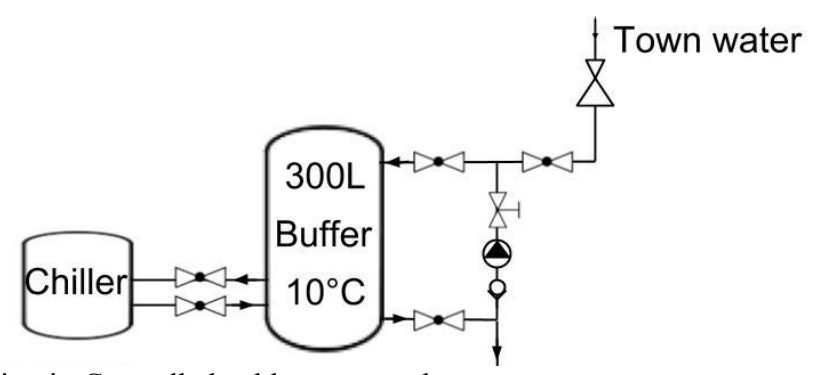

Fig. 3. Secondary circuit, Controlled cold water supply. 


\subsection{Local space heating}

Fan coils placed in a cold storage, kept at a fixed temperature, simulate the local space heating demand. Two fan coils at maximum fan speed can each subtract approximately $3 \mathrm{~kW}$, a third fan coil at maximum speed can subtract an additional $5 \mathrm{~kW}$. Each fan coil has three different fan speeds, each demanding a different flow rate of hot water (HW). Prior to the start of the test a fan speed of minimum, medium or maximum is selected. In initial test phases, fan speed is adjusted manually. Hydronicaly, the same dynamic valves are used as for the tapping cycles, with the minor adjustment of an additional electronic actuator. Using additional flow transmitters as feedback for the PLC, automated adjustments can be made to control the dissipation of heat. Future adjustments shall, if proven helpful, entail a stepless adjustment of fan speeds. This upgrade will allow us to continuously adjust the heat dissipation between $2 \mathrm{~kW}$ and $11 \mathrm{~kW}$, instead of selecting one of 27 combinations.

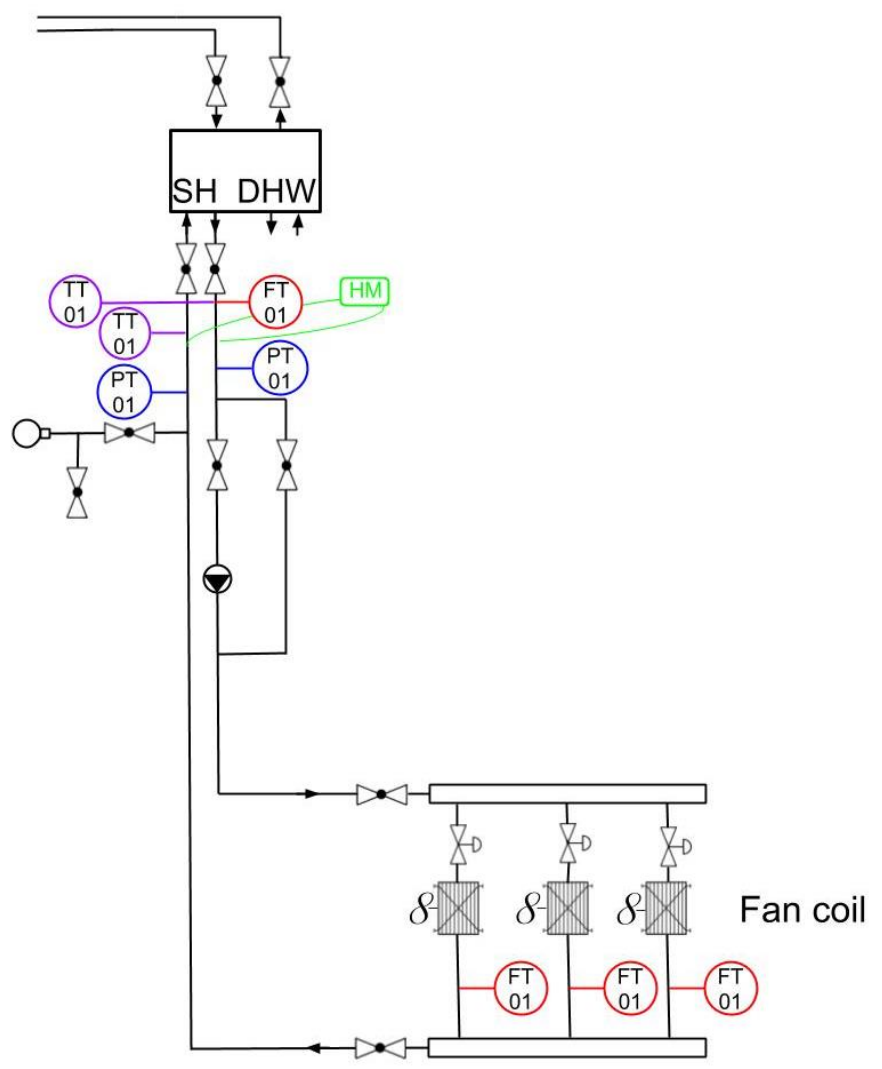

Fig. 4. Secondary circuit, Local space heating. 


\section{Test procedure}

As stated previously a test method has been standardized in NBN EN 13203 for testing gas fired appliances. For space heating ( $\mathrm{SH}$ ) the existing standard (test at $30 \%$ and $100 \%$ nominal load) provides little insight in the dynamic behaviour of the heat generator.

Also how a heat generator addresses a DHW demand combined with a heat demand for $\mathrm{SH}$, is not yet described. For this reason, a new method was developed. In general, the test procedures can be partitioned in two segments. One being assessments of performance of hot water deliveries and the other being assessment of energy consumption. Basically still referring to NBN EN 13203-1 [5] and 2 [6], respectively, but with the addition of a withdrawal of energy for space heating. An additional and highly influential parameter is the temperature regime at which the central supply works. The required electrical energy for the controller of the substation is also logged using a Beckhoff component.

\subsection{Assessments of performance of hot water deliveries}

An assessment of performance of hot water delivery can be described as how the unit reacts temperature wise and timewise to different variations in boundary conditions. Two main directions can be investigated. First maintaining a constant withdrawal of SH and allowing the DHW to vary, secondly, maintaining DHW and adjusting the heat dissipation. Investigating the units behaviour i.e., temperature fluctuations in $\mathrm{SH}$, required heat-up time for DHW and its ability to uphold this temperature is a straight forward indication of the units comfort capacity. In order to make a correct assumption, different temperature regimes of the primary circuit also need to be included as a boundary condition. Influenced by the manufacturer's control strategy and individually determined for each test case, flexibility towards lower temperatures in the central supply is investigated.

\subsubsection{Fixed SH, varying $\mathrm{DHW}$}

Based on a test prescribed towards independent test laboratories it is of interest that the return temperature of the central system remains above a certain threshold. In this case a central supply of $80^{\circ} \mathrm{C}$ and a return of $30^{\circ} \mathrm{C}$ with a maximum of $40^{\circ} \mathrm{C}$ needs to be maintained. The required power to dissipate is, prior to test, adjusted accordingly. A test sequence exists of doubling the flow rate at the DHW supply every minute until reaching the maximum applicable flowrate provided by the manufacturer, followed by halving the flow rate every minute until zero. This for no longer than seven minutes. The test sequence to impose is repeated for four times over the course of $24 \mathrm{~h}$.

A revision to this procedure is to use a fixed value for heat dissipation instead of a 'fixed' return temperature. To determine this value a typical apartment dwelling has been chosen in which the living room, bath room and bedroom are being heated. With an extreme outside temperature of $-8^{\circ} \mathrm{C}$, a required power of $2,5 \mathrm{~kW} ; 1 \mathrm{~kW}$ and $0,5 \mathrm{~kW}$ results in a total decrease of $4 \mathrm{~kW}$ to be withdrawn from the fan coils [7]. Test procedures described in NBN EN 13203-1 are applied and all data is logged for $24 \mathrm{~h}$. 


\subsubsection{Fixed DHW, varying $\mathrm{SH}$}

The same principle stands as with previous methods, but instead of an oft-recurring condition, being tapping of DHW, a slower les frequent occurring of added temperature shock by extra heat dissipation is investigated. Essentially adding a room to be heated or raising the requested room temperature. Though not the most acclaimed results, they can teach us more about the behaviour of the applied priority regulation of the unit and thereby its comfort level.

\subsection{Assessment of energy consumption}

As the assessment of performance teaches us how the unit reacts comfort wise, the assessment of energy consumption teaches us how efficient the unit is. Similar to NBN EN 13203-2, a tapping profile of different energy withdrawals is applied to the substation's DHW supply, as stated in 1.2. These tapping profiles are designed to match behaviour of certain sized families and dwellings and are divided starting from small (S) to four times extra-large (4XL). In a certifying environment it is the choice of the manufacturer to determine which size pattern to apply. In a research environment different variations are put to test.

By measuring input and output heat, efficiency and heat losses of the unit can be determined. By either choosing to apply SH, a prediction of summer and winter efficiency can be determined.

\subsection{Cold start}

A final assessment to be made is how the unit reacts from a cold start, albeit through comfort or efficiency. Imagine coming home from a long holiday and immediately wanting to heat up the house and take a shower at the same time. What will receive priority, how will return temperatures react, how fast can the unit heat up and what is its efficiency during warmup?

\section{Conclusion}

The construction and provision of considered test facility proves to fill up a highly anticipated gap in the research fields as well as with manufacturers.

A lab set-up is developed, which is able to simulate a high number of variations in control strategies and district heating lay-outs. Besides this, the set-up allows comparison with local heat generators.

A number of test procedures are evaluated which can be implemented to gather realistic data on dynamic behaviour of substations, allowing use in simulations.

Comparable studies will not only result in more energy efficient units, more appropriate dimensioning and selecting will result in more energy efficient buildings together with more accurate building simulation programs. Combined with a well dimensioned and finetuned Thermal Energy Storage tank (TES) these improvements will allow for a far more energy efficient implementation of hybrid configurations with renewable energy sources, especially for individual district heating systems. 


\section{References}

1. EC, "Horizon 2020 Work Programme: Secure, clean and efficient energy" (2015)

2. D. Connolly, H. Lund, B.V. Mathiesen, S. Werner, B. Möller, U. Persson, T. Boermans, D. Trier, P.A. Ostergaard, S. Nielsen, Ener. Pol. 65 (2014)

3. M. A. Ancona, M. Bianchi, L. Branchini, F. Melino, Ener. Proc. 45 (2014).

4. U. Persson, B. Möller, S. Werner, Ener. Pol. 74 (2014)

5. nbn, "NBN EN 13203-1: Gas fired domestic appliances producing hot water - Part 1: Assessment of performance of hot water deliveries," 1 (2015)

6. nbn, "NBN EN 13203-2: Gas-fired domestic appliances producing hot water - Part 2: Assessment of energy consumption," 2 (2015)

7. F. Van Riet, H. El Khaoui, F. Hulsbosch, G. Steenackers, and I. Verhaert,. "Exploring the novel software Hysopt : a comparison of hydronic heat distribution systems of an apartment building", REHVA Aalborg (2016) 